\title{
Numerical Study of Cross Diffusion Effects on Double Diffusive Convection with Lattice Boltzmann Method
}

\author{
Xiaomei $\mathrm{Yu}^{1}$, Zhaoli Guo ${ }^{1}$, and Baochang $\mathrm{Shi}^{2, \star}$ \\ ${ }^{1}$ State Key Laboratory of Coal Combustion, \\ Huazhong University of Science and Technology, \\ Wuhan 430074 P.R. China \\ yuxiaomei_hust@126.com, \\ zlguo@mail. hust.edu.cn \\ ${ }^{2}$ Department of Mathematics, \\ Huazhong University of Science and Technology, \\ Wuhan 430074 P.R. China \\ sbchust@126.com
}

\begin{abstract}
A lattice Boltzmann model is proposed to asses the impact of variable molecular transport effects on the heat and mass transfer in a horizontal shallow cavity due to natural convection. The formulation includes a generalized form of the Soret and Dufour mass and heat diffusion (cross diffusion) vectors derived from non-equilibrium thermodynamics and fluctuation theory. Both the individual cross diffusion effect and combined effects on transport phenomena are considered. Results from numerical simulations indicate that Soret mass flux and Dufour energy flux have appreciable effect and sometimes are significant. At the same time, the lattice Boltzmann model has been proved to be adequate to describe higher order effects on energy and mass transfer.
\end{abstract}

Keywords: lattice Boltzmann model; Soret effect; Dufour effect; Natural convection.

\section{Introduction}

Transport phenomena and thermo-physical property in fluid convection submitted to the influence of thermal and concentration horizontal gradients arise in many fields of science and engineering. The conservation equations which describe the transport of energy and mass in these fluid systems are well developed [1-3]. The energy flux includes contributions due to a temperature gradient (Fourier heat conduction), concentration gradient(Dufour diffusion) and a term which accounts for the energy transport as a results of each species having different enthalpies (species interdiffusion). The mass flux consists of terms due

\footnotetext{
^ Corresponding author.
} 
to a concentration gradient (Fickian diffusion), temperature gradient (Soret diffusion), Pressure gradient (pressure diffusion) and a term which accounts for external forces affecting each species by a different magnitude. However, most studies concerned on transport phenomena only considered the contributions due to Fourier heat conduction and Fickian diffusion.

The Soret mass flux and Dufour energy flux become significant when the thermal diffusion factor and the temperature and concentration gradients are large. Actually, Rosner [4] has stressed that Soret diffusion is significant in several important engineering applications. Similarly, Atimtay and Gill [5] have shown Soret and Dufour diffusion to be appreciable for convection on a rotating disc. An error as high as $30 \%$ for the wall mass flux is introduced when the Soret effect is not accounted for. Of particular interest, crystal growth from the vapor is sometimes carried out under conditions conductive to Soret and Dufour effects. As greater demands are made for tighter control of industrial processes, such as in microelctromechanical systems (MEMS) [6], second-order effects such as Soret and Dufour diffusion may have to be included.

In regard to actual energy and mass transport in double diffusive convection fluid systems which include Soret and Dufour cross diffusion effect, little, if any, complete and detailed work has been done. However, a few studies have considered the convection, within a vertical cavity, induced by Soret effects. The first study on this topic is due to Bergman and Srinivasan [7]. Their numerical results indicate that the Soret-induced buoyancy effects are more important when convection is relatively weak. The particular case of a square cavity under the influence of thermal and solutal buoyancy forces, which are opposing and of equal intensity, has been investigated by Traore and Mojtabi [8]. The Soret effect on the flow structures was investigated numerically. The same problem was considered by Krishnan [9] and Gobin and Bennacer [10] for the case of an infinite vertical fluid layer. The critical Rayleigh number for the onset of motion was determined by these authors. More recently, Ouriemi et al. [11] considered the case of a shallow layer of a binary fluid submitted to the influence of thermal and concentration horizontal gradients with Soret effects. Moreover, few studies have been concerned with the Soret and Dufour effects at the same time. Weaver and Viskanta numerically simulates these effects in a cavity [12]. Malashetty et al.[13] have performed an stability analysis of this problem. Because of the limited number of studies available, the knowledge concerning the influence of these effects on the heat and mass transfer and fluid flow is incomplete.

The lattice Boltzmann method (LBM) is a new method for simulating fluid flow and modeling physics in fluids. It has shown its power and advantages in wide range of situations for its mesoscopic nature and the distinctive computational features $[14,15,16]$. Contrary to the conventional numerical methods, the LBM has many special advantages in simulating physical problems. The objective of the present study is to develop an effective lattice Boltzmann model to examine the influences and the contributions of the Soret and Dufour effects on the natural convection with simultaneous heat and mass transfer across a horizontal shallow cavity. Based on the idea of the double distribution function 
models for a fluid flow involving heat transfer, a generalized lattice Boltzmann model is proposed to solve the control equations with Soret and Dufour effects.

\section{The Lattice Boltzmann Method for the Formulation of the Problem}

The configuration considered in this study is a horizontal shallow cavity, of width $H$ and length $L$. The top and bottom end walls were assumed to be adiabatic and impermeable to heat and mass transfer while the boundary conditions along the right and left side walls were subjected by Dirichlet conditions. Considering the Soret effects and Dufour effects, the dimensionless incompressible fluid equations for the conservation of mass, momentum, solutal concentration, and temperature, with the inclusion of the Boussinesq approximation for the density variation, are written as

$$
\begin{array}{r}
\nabla \cdot \mathbf{u}=0, \\
\frac{\partial \mathbf{u}}{\partial t}+\mathbf{u} \cdot \nabla(\mathbf{u})=-\nabla p+\left(\frac{P r}{R a}\right)^{1 / 2} \nabla^{2} \mathbf{u}+\operatorname{Pr}(T+\varphi C), \\
\frac{\partial T}{\partial t}+\mathbf{u} \cdot \nabla T=\frac{1}{\sqrt{R a p r}}\left(\nabla^{2} T+D_{C T} \nabla^{2} C\right), \\
\frac{\partial C}{\partial t}+\mathbf{u} \cdot \nabla C=\frac{1}{L e \sqrt{\operatorname{Rapr}}}\left(\nabla^{2} C+S_{T C} \nabla^{2} T\right)
\end{array}
$$

along with the boundary conditions where $\rho$ and $\mathbf{u}$ are the fluid density and velocity, respectively. $T$ is the temperature of the fluid.

The dimensionless variables are defined as

$$
\begin{gathered}
\mathbf{X}=\frac{\mathbf{X}}{H}, t=\frac{t \sqrt{R a}}{H^{2} / \alpha}, \mathbf{u}=\frac{\mathbf{u}}{(\alpha / H) \sqrt{R a}}, p=\frac{p}{\left(\alpha^{2} / H^{2}\right) R a}, T=\frac{T-T_{0}}{\Delta T}, \\
C=\frac{C-C_{0}}{\Delta C}, D a=\frac{K}{H^{2}}, \operatorname{Pr}=\frac{\nu}{\alpha}, L e=\frac{\alpha}{D}, R a=\frac{g \beta_{T} \Delta T H^{3}}{\nu \alpha}, \varphi=\frac{\beta_{C} \Delta C}{\beta_{T} \Delta T}
\end{gathered}
$$

Here, $\nu, \alpha, D$ are the kinematic viscosity, thermal diffusivity and diffusion coefficient,respectively. The remaining notation is conventional. From the above equations it is observed that the present problem is governed by the thermal Rayleigh number $R a$, buoyancy ratio $\varphi$, Lewis number Le, Prandtl number $P r$, Dofour factor $D_{C T}$ and Soret factor $S_{T C}$. In this paper, we concerned on the effect of Soret and Dufour factor. So other dimensionless variables will be kept at certain values.

Based on the idea of TLBGK model in [17], we take the temperature and concentration as passive scalar. Then the control equations of velocity, temperature and concentration can be treated individually. The evolution equation for the velocity field is similar to Ref. [18]

$$
\begin{array}{r}
f_{i}\left(\mathbf{x}+\mathbf{c}_{i} \Delta t, t+\Delta t\right)-f_{i}(\mathbf{x}, t)=-\frac{1}{\tau}\left(f_{i}(\mathbf{x}, t)-f_{i}^{e q}(\mathbf{x}, t)\right) \\
+\Delta t F_{i}(\mathbf{x}, t) \quad i=0, \ldots, b-1,
\end{array}
$$


where $\mathbf{c}_{i}$ is the discrete particle velocity, $F_{i}$ represents the force term, $f_{i}(\mathbf{x}, t)$ is the distribution function (DF) for the particle with velocity $\mathbf{c}_{i}$ at position $\mathbf{x}$ and time t, $\Delta t$ is the time increment. $\tau$ is the nondimensional relaxation time and $f_{i}^{(e q)}$ is the equilibrium distribution function (EDF). The EDF must be defined appropriately such that the mass and momentum are conserved and some symmetry requirements are satisfied in order to describe the correct hydrodynamics of the fluid. In the $D n Q b[19]$ models, the EDF is now defined as

$$
\begin{gathered}
f_{i}^{(e q)}=\alpha_{i} p+\omega_{i}\left[\frac{\mathbf{c}_{i} \cdot \mathbf{u}}{c_{s}^{2}}+\frac{\mathbf{u u}:\left(\mathbf{c}_{i} \mathbf{c}_{i}-c_{s}^{2} \mathbf{I}\right)}{2 c_{s}^{4}} .\right. \\
F_{i}=\frac{\left(\delta_{i 2}+\delta_{i 4}\right)}{2 c^{2}} \mathbf{c}_{i} \operatorname{Pr}(T+\varphi C)
\end{gathered}
$$

where $\omega_{i}$ is the weight and $c_{s}$ is the sound speed. Both $\omega_{i}$ and $c_{s}$ depend on the underlying lattice. Take the D2Q9 model for example, the discrete velocities are given by $\mathbf{c}_{0}=0$, and $\mathbf{c}_{i}=\lambda_{i}\left(\cos \theta_{i}, \sin \theta_{i}\right) c$ with $\lambda_{i}=1, \theta_{i}=(i-1) \pi / 2$ for $i=1-4$, and $\lambda_{i}=\sqrt{2}, \theta_{i}=(i-5) \pi / 2+\pi / 4$ for $i=5-8$, and $c_{s}=c / \sqrt{3}$. $\omega_{0}=4 / 9$ and $\alpha_{0}=-4 \sigma / c^{2}, \omega_{i}=1 / 9$ and $\alpha_{i}=\lambda / c^{2}$ for $(i=1,2,3,4), \omega_{i}=1 / 36$ and $\alpha_{i}=\gamma / c^{2}$ for $(i=5,6,7,8)$, respectively. Here, $\sigma=\frac{5}{12}, \lambda=\frac{1}{3}, \gamma=\frac{1}{12}$ is set [20] with best computation effects.

The fluid velocity $\mathbf{u}$ and pressure $p$ are defined by the $\mathrm{DF} f_{i}$

$$
\mathbf{u}=\sum_{i} \mathbf{c}_{i} f_{i}=\sum_{i} \mathbf{c}_{i} f_{i}^{(e q)}, \quad p=\frac{c^{2}}{4 \sigma}\left[\sum_{i \neq 0} f_{i}-\frac{2}{3} \frac{|\mathbf{u}|^{2}}{c^{2}}\right]
$$

Through the Chapman-Enskog expansion, the Eqs.(1),(2) can be obtained. The kinetic viscosity are given by $\left(\frac{P r}{R a}\right)^{1 / 2}=\frac{(2 \tau-1)}{6} c^{2} \Delta t$.

Similarly, the LBGK equations for temperature and concentration fields with D2Q5 lattice are written as follows,

$$
\begin{aligned}
& T_{i}\left(\mathbf{x}+\mathbf{c}_{i} \Delta t, t+\Delta t\right)-T_{i}(\mathbf{x}, t)=-\frac{1}{\tau_{T}}\left[T_{i}(\mathbf{x}, t)-T_{i}^{(0)}(\mathbf{x}, t)\right] \\
& S_{i}\left(\mathbf{x}+\mathbf{c}_{i} \Delta t, t+\Delta t\right)-S_{i}(\mathbf{x}, t)=-\frac{1}{\tau_{S}}\left[S_{i}(\mathbf{x}, t)-S_{i}^{(0)}(\mathbf{x}, t)\right]
\end{aligned}
$$

where $T_{i}$ and $S_{i}$ are the DF for the temperature field and concentration field, respectively. $\tau_{T}$ and $\tau_{S}$ are relaxation time corresponding to temperature DF and concentration DF trend to equilibrium. Just like the thoughts of constructing generalized LBGK model for Burgers equation [21], the EDF of temperature and concentration are defined as

$$
\begin{aligned}
T_{i}^{(0)} & =\frac{T}{4}\left[1-d_{0}+2 \frac{\mathbf{c}_{i} \cdot \mathbf{u}}{c^{2}}\right]+\frac{D_{S T} S\left(1-d_{0}\right)}{4}, \quad i \neq 0, \\
T_{0}^{(0)} & =T d_{0}-D_{S T} S\left(1-d_{0}\right), \quad i=0 . \\
S_{i}^{(0)} & =\frac{S}{4}\left[\left(1-l_{0}\right) \phi+2 \frac{\mathbf{c}_{i} \cdot \mathbf{u}}{c^{2}}\right]+\frac{S_{T S} T\left(1-l_{0}\right) \phi}{4}, \quad i \neq 0, \\
S_{0}^{(0)} & =S l_{0} \phi-S_{T S} T\left(1-l_{0}\right) \phi, \quad i=0 .
\end{aligned}
$$


The fluid temperature and concentration can be expressed by DFs,

$$
T=\sum_{i} T_{i}=\sum_{i} T_{i}^{(e q)}, S=\sum_{i} S_{i}=\sum_{i} S_{i}^{(e q)}
$$

It can be proved that the macroscopic temperature and concentration diffusion equations (3) and (4) can be recovered from $\operatorname{LBE}(9),(10)$. The corresponding thermal conduction coefficient, diffusion factor are

$$
\begin{gathered}
\frac{1}{\sqrt{\operatorname{RaPr}}}=\frac{c^{2}}{2}\left(\tau_{T}-\frac{1}{2}\right) \Delta t\left(1-d_{0}\right), \\
\frac{1}{\operatorname{Le} \sqrt{\operatorname{RaPr}}}=\frac{c^{2}}{2}\left(\tau_{T}-\frac{1}{2}\right) \Delta t\left(1-d_{0}\right) .
\end{gathered}
$$

Here, $d_{0}$ and $l_{0}$ are adjustable parameters. Obviously, the lattice Boltzmann model can simulate the double diffusive not only including Fourier heat conduction and Fickian diffusion but also second-order cross diffusion by choosing adequate parameters.

\section{Numerical Results and Discussion}

Using the lattice Boltzmann model, we simulate the Soret and Dufour effects in double diffusive due to temperature and concentration gradient. Since the Soret and Dufour effects are diffusive processes, the Rayleigh number should not too high in order to decrease the advective flux relative to the diffusive flux. Furthermore, the buoyancy ratio is set to be $\varphi=1$, which preserves the buoyancy forces induced by the thermal and solutal effects are of equal intensity. Simulation tests are conducted with varied Soret and Dufour factor and fixed Le and $\operatorname{Pr}$ number equal to 1 and 0.71, respectively. The lattice size is $128 \times 256$ with Ra equal to $10^{3}$.

Streamlines, isothermals and iso-concentration profiles varied with Dufour and Soret coefficients are shown in Fig. 1. The effect on mass, momentum and concentration transports made by varied cross diffusion coefficients can be observed obviously. The center velocity, local (averaged) Nusselt and Sherwood number on the left wall are showed in Fig. 2, Fig. 3 and Table 1 for detailed discussion, respectively. A positive (negative) value of Dufour and Soret coefficients decreases (increases) the value of the maximum velocity, and extends (reduces) the range of the velocity field. Moreover, Positive values for the Dufour and Soret coefficients yield solute concentrations below that of the free stream and so is the temperature, the opposite being also true. But when the Dufour coefficient is certain, the local Nusselt numbers decrease with Soret coefficients but local Sherwood number increase. While the Soret coefficient is certain, the local Nusselt numbers increse and the Sherwood number decrease instead. All curves show the expected behavior. 

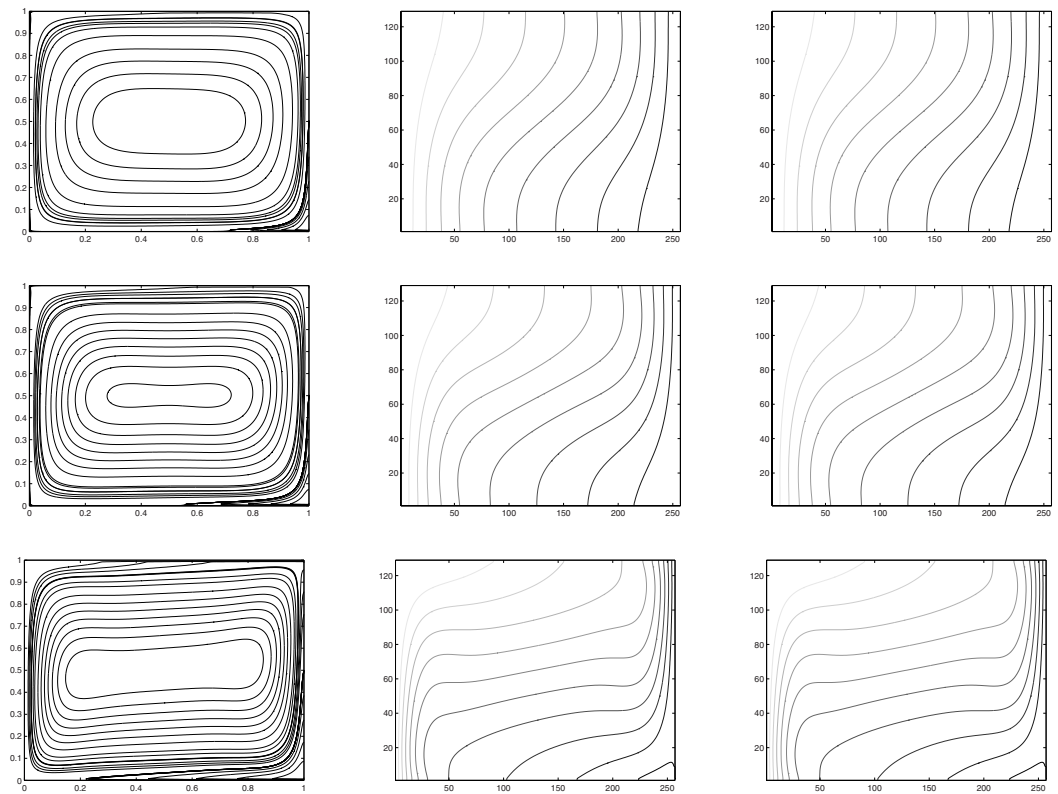

Fig. 1. Streamline, isothermals and iso-concentrations from left to right. From top to bottom, the Dufour and Soret coefficients are $(0,0),(0,0.5),(-0.5,-0.5),(-0.9,-0.9)$, respectively.
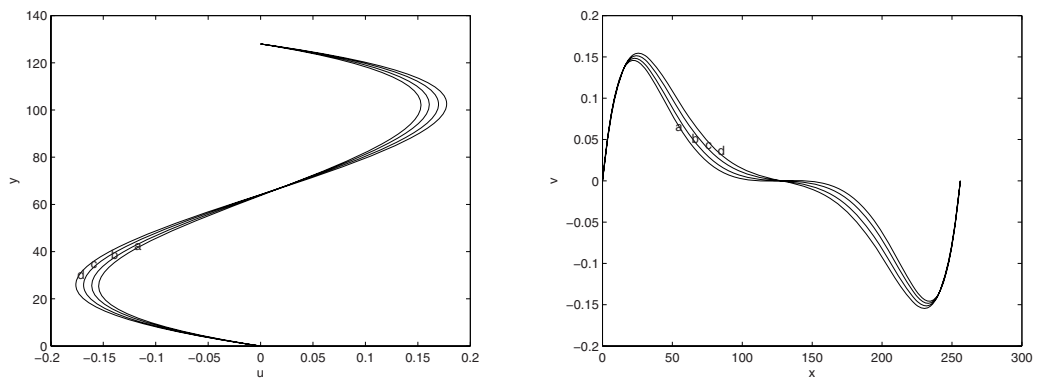

Fig. 2. The velocity components, $u$ and $v$, along the vertical and horizontal lines through the center at different cross diffusion numbers $\left(D_{C T}, S_{T C}\right)$ : a $=(0,-0.5)$; $\mathrm{b}=(0,0.0) ; \mathrm{c}=(0,0.5) ; \mathrm{d}=(0,0.9)$

Table 1. Averaged Nusselt and Sherwood number on the left wall veried with Soret and Dufour factors

\begin{tabular}{ccccccccccc}
\hline$(D, S)$ & $(0,-0.5)$ & $(0,0)$ & $(0,0.5)$ & $(0,0.9)$ & $-0.5,0)$ & $(0.5,0)$ & $(0.9,0)$ & $(-0.5,-0.5)$ & $(0.5,0.5)$ & $(0.9,0.9)$ \\
\hline$N u$ & 0.9570 & 0.7698 & 0.5608 & 0.3785 & 0.7465 & 0.7965 & 0.8200 & 1.0838 & 0.6532 & 0.6053 \\
\hline$S h$ & 0.7465 & 0.7698 & 0.7965 & 0.8200 & 0.9570 & 0.5608 & 0.3785 & 1.0838 & 0.6532 & 0.6053 \\
\hline
\end{tabular}



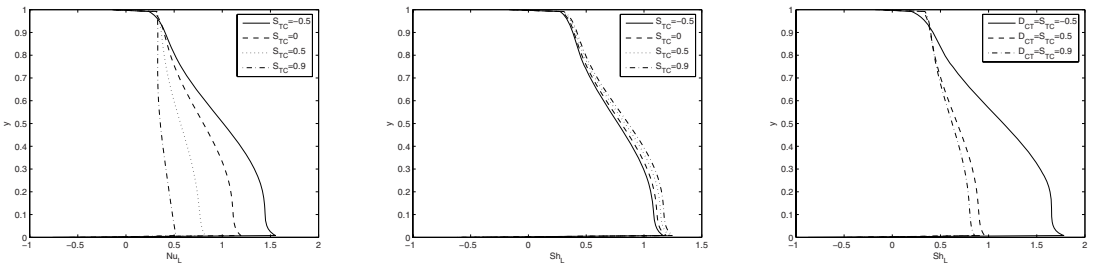

Fig. 3. Local Nusselt and Sherwood number on the left wall varied with cross diffusion numbers (The $D_{C T}$ is equal 0 at the left two figures)

\section{Summary}

In this paper,a lattice Boltzmann model is proposed and used to asses the Soret and Dufour effects on the heat and mass transfer in a horizontal shallow cavity due to natural convection. The proposed model is constructed in the double distribution function framework. Cross diffusion effects are studied under the buoyancy counteracting flows and augmenting flows in shallow cavity with the lattice Boltzmann model. The numerical results show that Soret and Dufour effects have contributions to mass and energy flux. Sometimes, it may appears to significant. Simulation results agree well with previous work. Moreover, it indicates that the lattice Boltzmann model is adequate to simulate molecular transport which even includes second-order effects. It seems that the lattice Boltzmann method is effective in describing higher order physical effects, which are important as the industry develops.

Acknowledgments. This work is supported by the National Basic Research Program of China (Grant No. 2006CB705804) and the National Science Foundation of China (Grant No. 50606012).

\section{References}

1. Hirshfelder J. O., Curtiss C. F., Bird R. B.: Molecular Theory of Gases and Liquids. Wiley, New York (1960)

2. Groot S. R., Mazur P.: Thermodynamics of Trreversible Processes. Dover, New York (1984)

3. Chapman S., Cowling T. G.: The Mathematical Theory of Non-Unifrom Gases. 3rd edn. Cambridge University, Cambridge (1970)

4. Rosner D. E.: Thermal (Soret) Diffusion Effects on Interfacial Mass Transport Rates. PhysicoChem. Hydordyn. 1 (1980) 159-185

5. Atimtay A. T., Gill W. N.: The Effect of Free Stream Concentration on Heat and Binary Mass Transfer with Thermodynamic Coupling in Convection on a Rotating Disc. Chem. Engng Commun. 34 (1985) 161-185

6. Karniadakis G. E., Beskok A.: Micro flows, Fundamentals and Simulation. Springer, Nw York (2001)

7. Bergman T. L., Srinivasan R.: Numerical Simulation of Soret-induced Double Diffusion in an Initiallly Uniform Concentration Binary Fluid. Int. J. Heat Mass Transfer 32 (1989) 679-687 
8. Traore Ph., Mojtabi A.: Analyse de l'effect soret en convection thermosolutale, Entropie 184/185 (1989) 32-37

9. Krishnan R.: A Numerical Study of the Instability of Double-Diffusive Convection in a Square Enclosure with Horizontal Temperature and Concentration Gradients, Heat transfer in convective flows. HTD ASME National Heat Transfer conference, vol 107. Philadelphia (1989)

10. Gobin D., Bennacer R.: Double-diffusion Convection in a Vertical Fluid Layer: Onset of the Convection Regime. Phys. Fluids 6 (1994) 59-67

11. Ouriemi M., Vasseur P., Bahloul A., Robillard L.: Natural Convection in a Horizontal Layer of a Binary Mixture. Int. J. Thermal Sciences 45 (2006) 752-759

12. Weaver J. A., Viskanta R.: Natural Convection Due to Horizontal Temperature and Concentration Gradients -2. Species Interdiffusion, Soret and Dufour Effects. Int. J. Heat Mass Transfer 34 (1991) 3121-3133

13. Malashetty M. S., Gaikward S. N., Effects of Cross Diffusion on Double Diffusive Convection in the Presence of Horizontal Gradients. Int. J. Engineering Science 40 (2002) 773-787

14. Benzi R., Succi S., Vergassola M.: The Lattice Boltzmann Equation: Theory and Applications. Phys. Report. 222 (1992) 145-197

15. Qian Y. H., Succi S., Orszag S.: Recent Advances in Lattice Boltzmann computing. Annu. Rev. Comp. Phys. 3 (1995) 195-242

16. Chen S. Y., Doolen G.: Lattice Boltzmann Method for Fluid Flows. Annu. Rev. Fluid Mech. 30 (1998) 329-364

17. Guo Z. L., Shi B. C., Zheng C. G.: A Coupled Lattive BGK Model for the Bouessinesq Equation. Int. J. Num. Meth. Fluids 39 (2002) 325-342

18. Deng B., Shi B. C., Wang G. C: A New Lattice-Bhatnagar-Gross-Krook Model for the Convection-Diffusion Equation with a Source Term. Chinese Phys. Lett. 22 (2005) 267-270

19. Qian Y. H., D'Humières D., Lallemand P.: Lattice BGK Models for Navier-Stokes Equation. Europhys. Lett. 17 (1992) 479-484

20. Guo Z. L., Shi B. C., Wang N. C., Lattice BGK model for incompressible NavierStokes Equation. J. Comput. Phys. 165 (2000) 288-306

21. Yu X. M., Shi B. C.: A Lattice Bhatnagar-Gross-Krook model for a class of the generalized Burgers equations. Chin. Phys. Soc. 15 (2006) 1441-1449 